\title{
Gene expression profile in newborn rat lungs after two days of recovery of mechanical ventilation
}

\author{
Valérie Dénervaud', Sandrine Gremlich ${ }^{1}$, Eliane Trummer-Menzi ${ }^{1}$, Johannes C. Schittny ${ }^{2}$ and Matthias Roth-Kleiner ${ }^{1}$
}

\begin{abstract}
BACKGROUND: Preterm infants having immature lungs often require respiratory support, potentially leading to bronchopulmonary dysplasia (BPD). Conventional BPD rodent models based on mechanical ventilation (MV) present outcome measured at the end of the ventilation period. A reversible intubation and ventilation model in newborn rats recently allowed discovering that different sets of genes modified their expression related to time after MV. In a newborn rat model, the expression profile $48 \mathrm{~h}$ after MV was analyzed with gene arrays to detect potentially interesting candidates with an impact on BPD development.
\end{abstract}

METHODS: Rat pups were injected P4-5 with $2 \mathrm{mg} / \mathrm{kg}$ lipopolysaccharide (LPS). One day later, MV with 21 or $60 \%$ oxygen was applied during $6 \mathrm{~h}$. Animals were sacrified $48 \mathrm{~h}$ after end of ventilation. Affymetrix gene arrays assessed the total gene expression profile in lung tissue.

RESULTS: In fully treated animals (LPS $+\mathrm{MV}+60 \% \mathrm{O}_{2}$ ) vs. controls, 271 genes changed expression significantly. All modified genes could be classified in six pathways: tissue remodeling/ wound repair, immune system and inflammatory response, hematopoiesis, vasodilatation, and oxidative stress. Major alterations were found in the MMP and complement system.

CONCLUSION: MMPs and complement factors play a central role in several of the pathways identified and may represent interesting targets for BPD treatment/prevention.

B ronchopulmonary dysplasia (BPD) is a chronic lung disease occurring in $~ 30 \%$ of preterm infants born less than $30 \mathrm{wk}$ of gestation (1). Its main risk factors include lung immaturity due to preterm delivery, mechanical ventilation (MV), oxygen toxicity, chorioamnionitis, and sepsis. The main feature is an arrest of alveolar and capillary formation (2). Models trying to decipher genes involved in the pathophysiology of BPD are mainly based on MV and oxygen application to young mammals with immature lungs of different species (3). In newborn rodent models, analyses of lung structure and gene and protein expression are performed for practical reasons directly at the end of MV (4-6). However, later appearing changes of gene expression might also have an impact on lung development and the evolution towards BPD and cannot be discovered by such models. Recently, we developed a newborn rat model of MV using an atraumatic (orotracheal) intubation technique that allows the weaning of the newborn animal off anesthesia and MV, the extubation to spontaneous breathing, and therefore allows the evaluation of effects of MV after a ventilationfree period of recovery (7). Indeed, applying this concept of atraumatic intubation by direct laryngoscopy, we recently were able to show significant differences between gene expression changes appearing directly after MV compared to those measured after a ventilation-free interval of $48 \mathrm{~h}$. Immediately after $\mathrm{MV}$, inflammation-related genes showed a transitory modified expression, while another set of more structurally related genes changed their expression only after a delay of $2 \mathrm{~d}$ (7). Lung structure, analyzed by conventional 2D histology and also by 3D reconstruction using synchrotron $\mathrm{x}$-ray tomographic microscopy revealed, $48 \mathrm{~h}$ after end of MV, a reduced complexity of lung architecture compared to the nonventilated rat lungs, similar to the typical findings in BPD. To extend these observations about late gene expression modifications, we performed with a similar model a full gene expression profile of lung tissue $48 \mathrm{~h}$ after the end of MV with either room air or $60 \%$ oxygen. Essentially, we measured changes in the expression of genes related to the MMPs and complement system which played a role in many of the six identified mostly affected pathways.

\section{RESULTS}

\section{Mechanical Ventilation Experiments and Weight Gain}

In preliminary experiments, we defined ventilation parameters in order to keep blood gas values within the physiologic range (Table 1). To measure rat pup's well-being during and after the ventilation episode, arterial blood was collected after 1 or $6 \mathrm{~h}$ of $\mathrm{MV}$, and blood gases were analyzed. All animals tested showed normal values of $\mathrm{pH}, \mathrm{PcO}_{2}, \mathrm{PO}_{2}$ (except for hyperoxia in animals ventilated with $60 \%$ oxygen; Table 2 ). Weight gain per day was of $15 \pm 4 \%$. There was no difference before and after the treatment in all groups. During the days of intervention, daily weight gain was slightly reduced with $6 \pm 6 \%$ in animals submitted to lipopolysaccharide (LPS) and MV.

\section{Gene Arrays}

Results of the gene array experiments were expressed as fold changes between the four groups: controls without LPS injection and ventilation (CTRL), LPS-injected (LPS), LPS-injected 
+ ventilated with room air (LMV), and LPS-injected + ventilated with $60 \%$ oxygen (LMVO) animals. Comparisons were made either step by step between the four groups (LPS vs. CTRL, LMV vs. LPS, LMVO vs. LMV), between LMVO and LPS, or overall between the full treatment and the control group (LMVO vs. CTRL). In the step by step analysis, 195 genes showed a statistically significant over or underexpression

Table 1. Ventilation parameters used in the mechanical ventilation experiments

\begin{tabular}{|c|c|c|c|}
\hline \multicolumn{4}{|l|}{ Ventilation parameters } \\
\hline \multicolumn{3}{|c|}{ Respiratory rate (per minute) } & 25 \\
\hline \multicolumn{3}{|l|}{ Inspiration-expiration ratio } & $1: 3$ \\
\hline \multicolumn{3}{|l|}{$\operatorname{PEEP}\left(\mathrm{cm} \mathrm{H}_{2} \mathrm{O}\right)$} & $1.5 \pm 0.6$ \\
\hline \multicolumn{3}{|l|}{ Pplat $\left(\mathrm{cm} \mathrm{H}_{2} \mathrm{O}\right)$} & $13.9 \pm 1.5$ \\
\hline \multicolumn{3}{|l|}{$\mathrm{PIP}\left(\mathrm{cm} \mathrm{H}_{2} \mathrm{O}\right)$} & $25.6 \pm 2.6$ \\
\hline \multicolumn{3}{|l|}{$\mathrm{MAP}\left(\mathrm{cm} \mathrm{H}_{2} \mathrm{O}\right.$} & $6.7 \pm 0.5$ \\
\hline \multicolumn{3}{|l|}{$\mathrm{FiO}_{2}$} & 60 or 21 \\
\hline \multicolumn{4}{|c|}{$\begin{array}{l}\text { Values are expressed as mean } \pm \text { SD from } 19 \text { independent animals. } \\
\text { FiO2, fraction of inspired oxygen; MAP, mean airway pressure; PEEP, positive end- } \\
\text { expiratory pressure; PIP, peak inspiratory pressure; Pplat: plateau pressure = pressure } \\
\text { measured at the end of inspiration and before start of expiration. }\end{array}$} \\
\hline Arterial blood gas values & $1 \mathrm{~h} \mathrm{FiO}{ }_{2} 0.60$ & $6 \mathrm{~h} \mathrm{FiO}_{2} 0.60$ & $6 \mathrm{~h} \mathrm{FiO}_{2} 0.21$ \\
\hline $\mathrm{PO}_{2}(\mathrm{~mm} \mathrm{Hg})$ & $152.5 \pm 24.4$ & $174.8 \pm 24.8$ & $71.4 \pm 6.7$ \\
\hline $\mathrm{PCO}_{2}(\mathrm{~mm} \mathrm{Hg})$ & $48.0 \pm 5.6$ & $47.1 \pm 4.3$ & $42.5 \pm 3.0$ \\
\hline $\mathrm{pH}$ & $7.39 \pm 0.03$ & $7.37 \pm 0.01$ & $7.34 \pm 0.00$ \\
\hline Glucose (mmol/l) & $6.9 \pm 1.3$ & $10.0 \pm 0.3$ & $8.7 \pm 4.1$ \\
\hline
\end{tabular}

Values are expressed as mean \pm SD from four independent animals per group. between the LPS and the CTRL group, 17 genes between the LMV and the LPS group and 11 between the LMVO and the LMV group. When analyzing the genes with a modified expression between LMVO and LPS (altogether 15 genes; Supplementary Table S1 online), three major gene expression patterns were observed across the three treatments (LPS, LMV, LMVO): a constant decrease between the LPS to LMV and the LMV to LMVO group (in $40 \%$ of the genes), a constant increase (in 20\%), or only an effect of MV without any effect of oxygen (27\%).

The overall comparison between the LMVO and the CTRL group retrieved 271 genes with statistically significant expression changes (208 upregulated and 63 downregulated; Supplementary Table S2 online). Classification of these genes according to their expression profile across treatment groups defined six major patterns (Figure 1). The first pattern represented genes with a progressive decrease in expression across treatments (5\% of genes; Figure 1a). The other five patterns all showed a first increase in gene expression probably due to LPS application (Figure 1b-f). In 38\% of these genes, no further effect of any additional treatment was observed (Figure 1d). In $22 \%$, an effect, either positive (16\%; Figure 1f) or negative (6\%; Figure 1b), could be attributed to MV without any further effect of oxygen. In $11 \%$ of the genes, an increased expression was observed due to oxygen with (in 5\%; Figure 1c) or without (in 6\%; Figure 1e) an associated downregulatory effect of MV.

Analyzing these genes through Metacore software, we identified six pathway folders that were significantly altered. These folders included (i) the immune system response, (ii) the inflammatory response, (iii) hematopoiesis, (iv) vasodilation, (v) oxidative stress regulation, and (vi) tissue remodeling/ wound repair (Figure 2). a

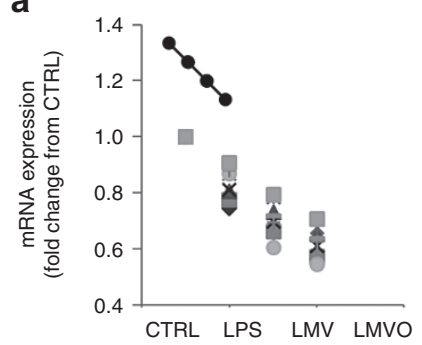

d

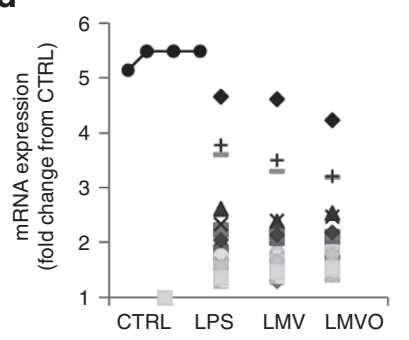

b

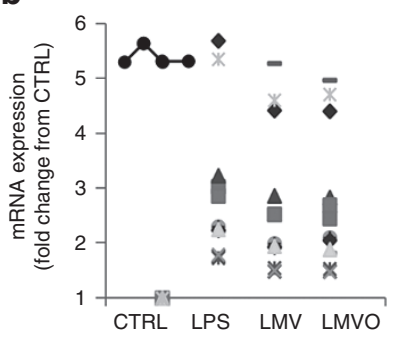

e

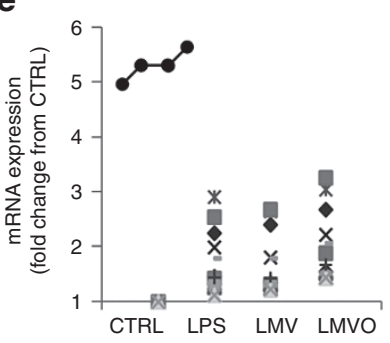

C

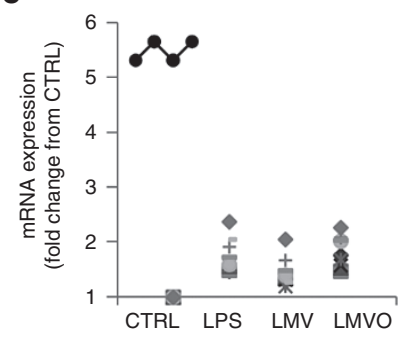

f

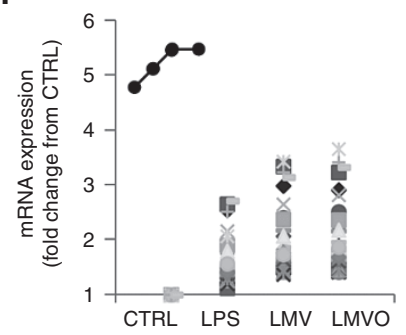

Figure 1. Principal gene expression profiles across the four treatment groups. Genes expressed $\geq 1.4$-fold higher in the LMVO group compared to the CTRL group with $P$ values $\leq 0.05$ were classified following their gene expression profiles across the different experimental groups. Only the principal profiles ( $\geq 5 \%$ of the genes with modified expression) are presented. The six graphs represent (a) $5 \%$, (b) $6 \%$, (c) $5 \%$, (d) $38 \%$, (e) $6 \%$, and (f) $16 \%$ of all significantly modified genes between control group and LMVO-group (LMVO: LPS + mechanical ventilation + $60 \%$ oxygen). The black and gray symbols indicate the different genes. The insets with the black line represent the common gene expression profiles across the four experimental groups. 


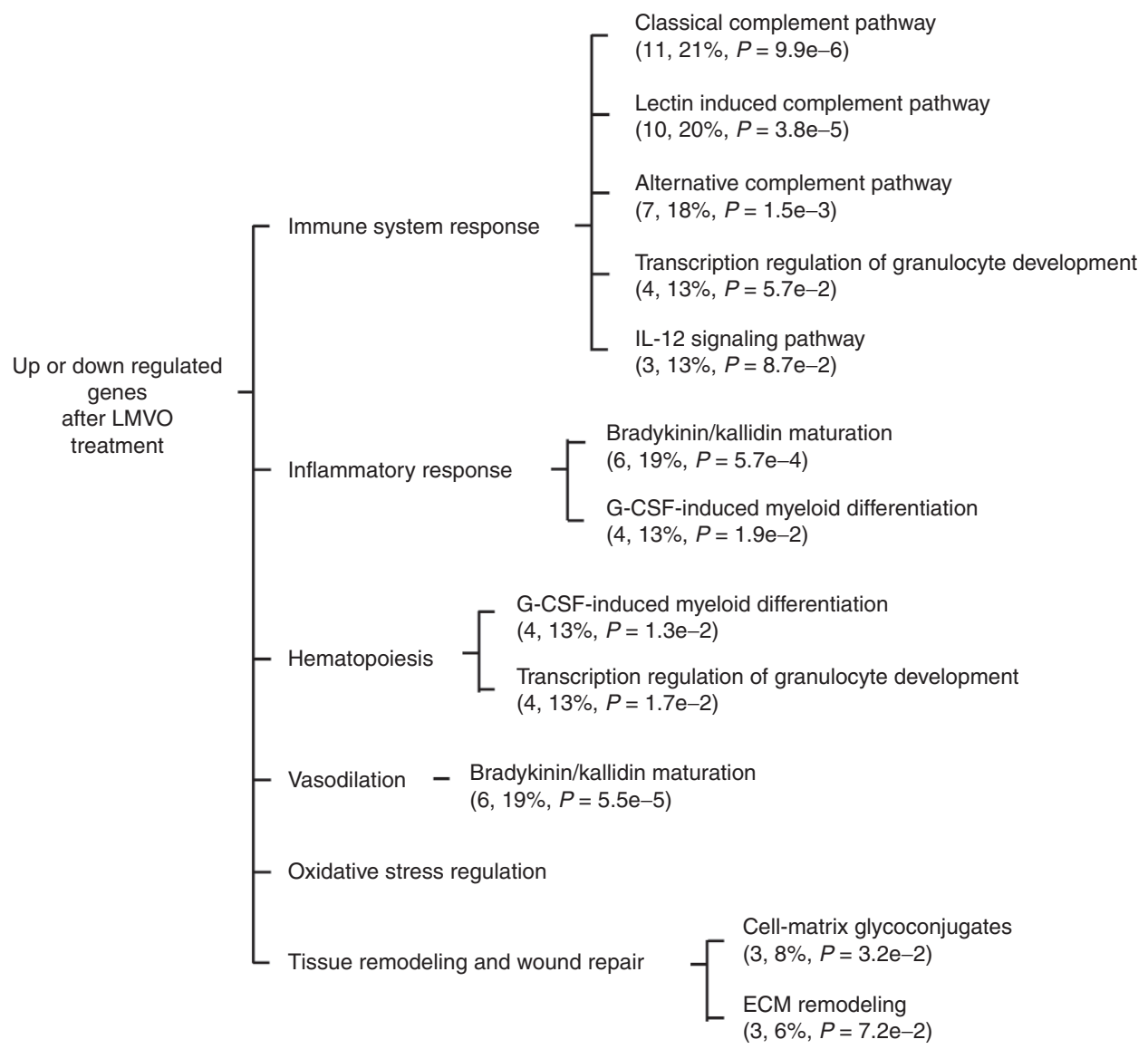

Figure 2. Pathway folders identified by Metacore software as being modified by LMVO treatment. Pathway maps and map folders modified by/in the modified gene expression profile. In brackets is the percentage of modified genes compared to the whole number of genes of the pathway, with the $P$ value rating their changes.

Pathways related to the immune system response included the three complement pathways (classical, lectin induced, and alternative) with an increased expression of complement components C1s, C1qa, C1qb, C3aR1, and C4-2 genes, the transcription regulation of granulocyte development with an increased expression of CD45/PTPRC (a signaling molecule), myeloblastin (a serine protease), and granulocyte colonystimulating factor (G-CSF) receptor genes, and IL-12 signaling pathway with an increased expression of IL-12 receptor b2, STAT4, and perforin, a cytolytic protein secreted by cytotoxic T-lymphocyte genes. Pathways related to the inflammatory response folder were essentially the bradykinin/kallidin maturation, with an increased expression of kininogen 1 gene, the precursor of the vasodilator bradykinin, and G-CSF-induced myeloid differentiation with an increased expression of integrin alpha M (Itgam) (a regulating molecule of leukocyte migration and adhesion), G-CSF receptor, myeloblastin, and lipocalin-2/ NGAL genes. Genes related to hematopoiesis included G-CSF-induced myeloid differentiation and the transcription regulation of granulocyte development. The vasodilation pathway folder highlighted the bradykinin/kallidin maturation pathway while the oxidative stress regulation pathway folder was related to glutathione metabolism, exemplified by the increased expression of glutathione-S-transferase GSTA5 which is responsible for the detoxification of the cell notably from products of oxidative stress. Finally, the tissue remodeling and wound repair pathway folder comprised cell matrix glycoconjugates and extracellular matrix (ECM) remodeling pathways. Cell matrix glycoconjugates were related to an increased expression of L-selectin gene, a cell adhesion molecule found essentially on lymphocytes, MMP9, and CCL5/ RANTES chemokine genes. ECM remodeling was related to an increase in MMP9 gene expression, in association with an increase in TIMP1 gene (tissue inhibitor of metalloproteinases) and a decrease in MMP12 gene expression. MMP9 and MMP12 are matrix metalloproteinases which mainly process gelatin and collagen IV-V (MMP9) and elastin, fibronectin, and collagen IV (MMP12).

Processes highlighted in the analysis of our results through Metacore software are listed in Table 3. These processes were linked to the immune system response, inflammatory response, hematopoiesis, iron transport, proteolysis (in relation with ECM remodeling or connective tissue degradation), and blood coagulation.

\section{Quantitative RT-PCR Verification}

In order to confirm gene array results, a series of candidate genes were evaluated by quantitative RT-PCR and compared to 
Table 3. Processes identified by Metacore software as being modified by LMVO treatment

\begin{tabular}{ll}
\hline Processes modified by mechanical ventilation treatment & $P$ value \\
\hline Inflammation_complement system & $2.4 \times 10^{8}$ \\
Chemotaxis & $3.4 \times 10^{7}$ \\
Cell adhesion_leukocyte chemotaxis & $6.2 \times 10^{6}$ \\
Inflammation_innate inflammatory response & $4.0 \times 10^{5}$ \\
Inflammation_kallikrein-kinin system & $2.3 \times 10^{4}$ \\
Immune response_phagocytosis & $1.1 \times 10^{3}$ \\
Immune response_antigen presentation & $1.5 \mathrm{e} \times 10^{3}$ \\
Inflammation_NK cell cytotoxicity & $1.5 \times 10^{3}$ \\
Immune response_BCR pathway & $1.9 \times 10^{3}$ \\
Cell adhesion_glycoconjugates & $5.2 \times 10^{3}$ \\
Immune reponse_Th17-derived cytokines & $5.5 \times 10^{3}$ \\
Inflammation_IL-2 signaling & $7.3 \times 10^{3}$ \\
Cell adhesion_platelet-endothelium-leukocyte interactions & $8.0 \times 10^{3}$ \\
Transport_iron transport & $8.7 \times 10^{3}$ \\
Inflammation_interferon signaling & $9.5 \times 10^{3}$ \\
Proteolysis_ECM remodeling & $1.3 \times 10^{2}$ \\
Proteolysis_connective tissue degradation & $1.4 \times 10^{2}$ \\
Blood coagulation & $1.9 \times 10^{2}$ \\
Proliferation_lymphocyte proliferation & $2.2 \times 10^{2}$ \\
Inflammation_neutrophil activation & $2.6 \times 10^{2}$ \\
Immune system_Thelper cell differentiation & $2.8 \times 10^{2}$ \\
Signal transduction_cholecystokinin signaling & $3.1 \times 10^{2}$ \\
Inflammation_amphoterin signaling & $4.5 \times 10^{2}$ \\
Immune response_phagosome in antigen presentation & $4.8 \times 10^{2}$ \\
\hline
\end{tabular}

the gene array data (Figure 3). For all of them, the gene expression changes observed by qRT-PCR were consistent with the gene array results.

\section{Proteins of Tissue Remodeling and Wound Repair Pathway Folder}

Some genes, highlighted both by pathways and processes, were further evaluated at the protein level: MMP9, MMP12, TIMP1, and lipocalin-2/NGAL (Figure 4). Indeed, MMP9-lipocalin-2 complex was $3.09 \pm 0.46$-fold higher in the LMVO group compared to the CTRL group $(P=0.01)$. MMP12 protein expression was $0.42 \pm 0.08$-fold lower in the LMVO compared to the CTRL group $(P=0.002)$ and lipocalin-2/NGAL $2.37 \pm 0.29$ fold higher in LMVO compared to CTRL group $(P=0.009)$. Conversely, TIMP1, whose mRNA expression was increased in the LMVO compared to the CTRL group, showed a lower protein expression in the LMVO group $(0.68 \pm 0.11$-fold vs. CTRL; $P=0.039$; Figure 4c).

\section{DISCUSSION}

To better understand the molecular pathways involved in the development of BPD, we recently developed a newborn rat model enabling us to analyze late effects of inflammation, oxygen, and MV. Thanks to an atraumatic intubation procedure performed under direct laryngoscopy, we were able to wean ventilated newborn rats off anesthesia and MV which allowed us then to perform outcome measurements after a chosen time period of recovery (7). In addition to the transitory increase of proinflammatory cytokines/chemokines measured in different studies immediately after MV and/or hyperoxia exposure in immature lungs $(4,8,9)$, our previous study demonstrated changes in MMP9 and tropoelastin mRNA expression only as a delayed reaction $48 \mathrm{~h}$ after the ventilation episode (7). This indicated a second wave of gene regulation that could correspond to mechanisms occurring in premature infants developing BPD. Therefore, we analyzed the complete gene expression profile in lungs of newborn rats $48 \mathrm{~h}$ after ventilation using gene arrays.

Tissue remodeling/wound repair was found to be one of the mostly affected pathway folders. It consists of a network of proteases/antiproteases including MMP9, MMP12, TIMP1, MMP8, secretory leukocyte peptidase inhibitor, and ADAM metallopeptidase with thrombospondin type 1 motif, 5 (ADAMTS5). The function of MMP9, also called gelatinase B, has been studied in lung development and lung inflammatory diseases such as BPD $(3,10)$. Levels of MMP9 are increased in animal models of BPD (11-13). MMP9 has been shown to be involved in ventilator- and oxygen-induced lung injury and has a potential for destruction of lung matrix and basement membrane (14). MMP9 knockout mice showed increased survival and less important modifications of lung structure after hyperoxia exposure compared to wild-type animals (15). Furthermore, MMP9/TIMP1 ratio has been shown to be elevated in tracheal aspirates of preterm infants who developed BPD $(16,17)$. In our experiments, changes in MMP9 protein expression were synergistic with changes in other genes and proteins of the same pathway. Indeed, the increased MMP9 expression in LMVO (LPS-injected animals + ventilation with $60 \%$ oxygen) compared to CTRL group was associated to a decrease in TIMP1 expression, its principal inhibitor. A further increase of MMP9 activity could be expected from the observed increase in lipocalin-2/NGAL protein, which is able to bind MMP9 and inhibit its degradation. The increase in the complex formed by MMP9 and lipocalin-2 in the LMVO group was confirmed by MMP9 western blots, under the form of a $125 \mathrm{kDa}$ band (Figure 4) $(18,19)$. Lipocalin-2/NGAL has been shown to be increased in serum and bronchoalveolar lavage fluid of premature infants developing BPD, and its level was associated with the risk of BPD development in a multivariate analysis $(20,21)$. Taken together, these results suggest a combined effect toward an increase of MMP9 activity in ventilated immature lungs.

In our previous experiments, we had shown that elastin protein expression was unchanged in ventilated lungs after $48 \mathrm{~h}$, whereas the mRNA expression of tropoelastin gene was decreased (7). Several enzymes control elastin metabolism, like neutrophil elastase, alpha-1 antitrypsin, lysyl oxidase or the elastin binding protein. In our gene array, the macrophage elastase MMP12 was downregulated in the LMVO group, also 
a

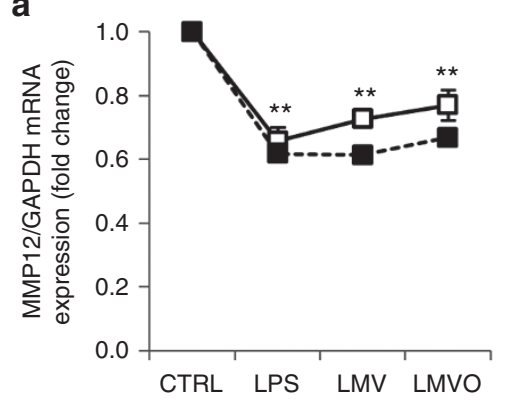

d

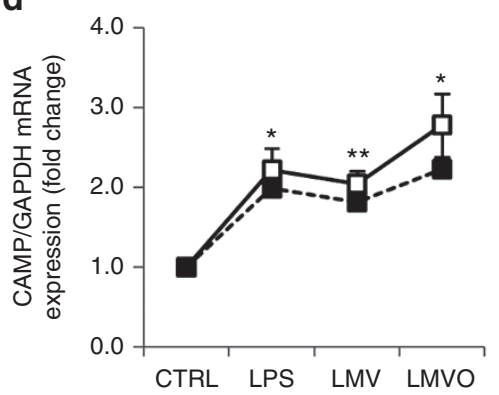

b

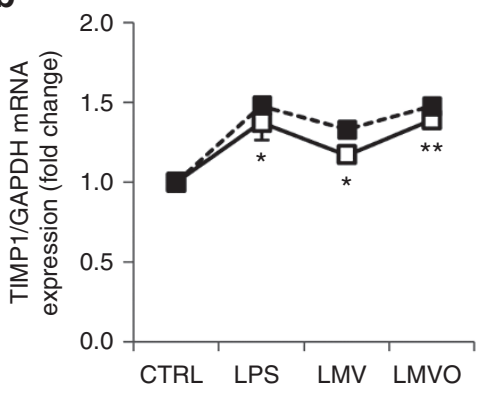

e

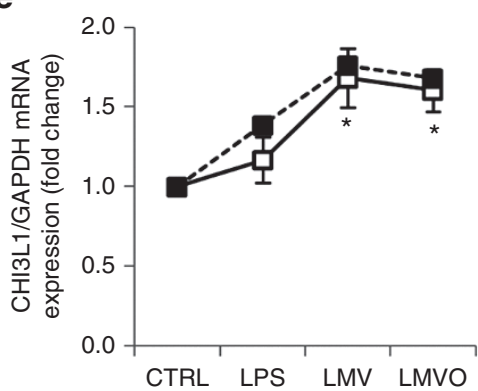

C

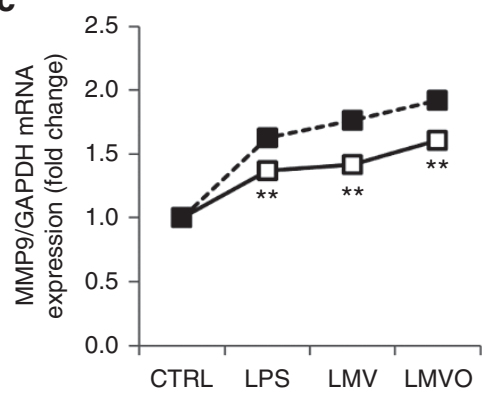

f

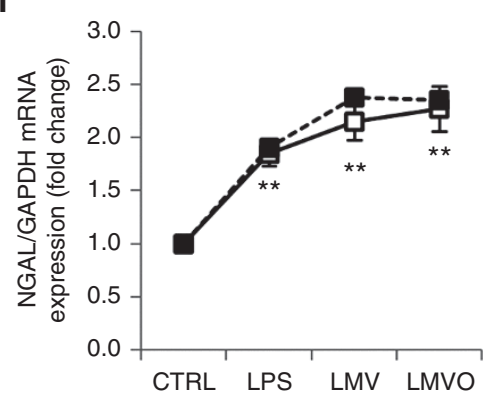

g

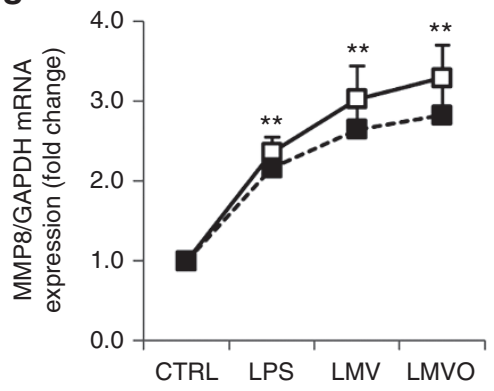

h

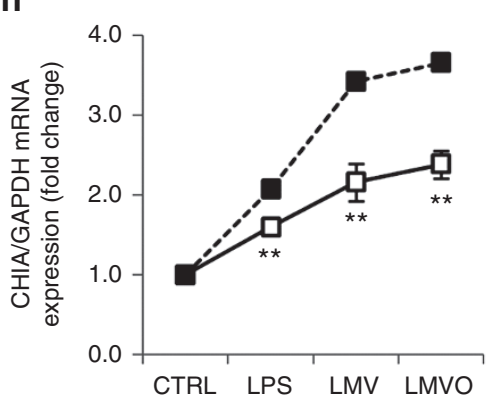

Figure 3. Quantitative RT-PCR of a series of candidate genes and comparison with gene array results. A series of genes were verified by qRT-PCR on all lung samples of the four treatment groups. In black squares, the gene array results, and in white squares, the qRT-PCR results $(n=3)$. (a) MMP12, (b) TIMP1, (c) MMP9, (d) CAMP (cathelicidin antimicrobial peptide), (e) CHI3L1 (chitinase 3-like 1), (f): NGAL (lipocalin 2), (g) MMP8, (h) CHIA (chitinase, acidic). The expression of each gene of interest is normalized by GAPDH housekeeping gene expression. Quantitative RT-PCR results are presented as mean \pm SEM. ${ }^{*} P<0.05 ;{ }^{* *} P<0.01$ as analyzed by Student's $t$-test against the CTRL value.

confirmed by a decreased protein expression. Neutrophil and macrophage elastases have been shown to have a complex interaction. Neutrophil elastase is able to cleave pro-MMP12 releasing mature MMP12 and to inactivate TIMP1 by cleavage. MMP12 itself inactivates alpha- 1 antitrypsin (neutrophil elastase inhibitor) by cleavage and increases the chemotactic signals for neutrophils $(22,23)$. Both neutrophil and macrophage elastases have been associated with lung injury (24). Our results confirm recently published experiments showing that prolonged ventilation with $40 \%$ oxygen increased MMP9 and elastase activity, resulting in elastin degradation and emphysematous alveoli, similar to findings in BPD. Treatment with the elastase inhibitor elafin showed promising reduction of these alterations $(6,25)$.

Similar to pediatric with acute respiratory distress syndrome (ARDS) patients, in which higher MMP9 and MMP8 activity in tracheal aspirates was correlated with a longer need for MV (26), an increase in MMP8 in bronchoalveolar lavage fluid and lung tissue of rodents was observed after exposure to high-pressure ventilation or prolonged ( $48 \mathrm{~h}$ ) high oxygen exposure (27). Inactivation of MMP8 by a selective inhibitor resulted in less lung injury in a model of MV (28). Therefore, the fact that our gene array experiments identified an increase of MMP8 gene expression 48 h after MV might also point to MMP8 as an interesting target to reduce negative effects of MV and oxygen.

MMPs have been discovered due to their role in ECM degradation. Since then, they have been assigned many other roles notably in inflammation, innate immunity, embryogenesis, wound healing, cell invasion, and angiogenesis (29-32). Indeed, MMPs are able to alter the activity of other proteases, liberate ECM-bound bioactive fragments and proteins (e.g., vascular endothelial growth factor or transforming growth factor- $\beta$ ), activate or inactivate many chemokines, exert membrane shedding activity, convert matrix proteins in bioactive signaling molecules, release active proteins from inhibitory complexes (e.g., vascular endothelial growth factor), or expose sites with biological activity by proteolytic cleavage 
of ECM proteins (30-32). This indicates that substrates of MMPs are much more numerous than initially thought. For example, MMP9, which is secreted from neutrophils following IL-8 stimulation, was shown to cleave IL- 8 chemokine and to increase its chemoattractant potency by 10 -fold. MMP8 is required for CXCL6 neutrophil-recruiting chemokine activation. Finally, MMP12 plays a role in the regulation of the immune response duration by cleaving and inactivating a large number of chemokines to stop neutrophil/macrophage recruitment at the site of infection, by direct inhibition of the production of some proinflammatory cytokines and by disrupting the attachment of chemokines to the ECM or cell membrane $(31,33)$. Therefore, the observed gene and protein expression changes in the MMP family may have an important impact on lung development and its deviation toward BPD by different mechanisms and pathways. Or in other words, regulating MMP activity might open new strategies to protect ventilated lungs from ventilator- and oxygen-induced lung injury.

Many of the observed gene expression changes were involved in pathways related to the immune system, the inflammatory response, and hematopoiesis. In our previous experiments, we had found that the cytokines/chemokines whose gene expression was increased immediately after MV had returned to basal values when evaluated $48 \mathrm{~h}$ after end of ventilation (7). Indeed, in the present study, we confirm that the cytokine gene expression was normalized by $48 \mathrm{~h}$. In fact, other genes than the cyto-/chemokines showed changes in gene expression leading to alteration of immune response and inflammation. In particular, we found that the immune system response was still or de novo stimulated at $48 \mathrm{~h}$ through the increased expression of many components of the complement system (C1s, C1qa, C1qb, while C3, C3aR, and C4-2). Several studies have shown a correlation between complement activation and ventilator-induced lung injury. Notably in rats, MV was shown to stimulate complement activity and its extravasation into lung tissue resulting in increased vascular permeability, which is one of the hallmarks of ventilator-induced lung injury (34). Takahashi et al. (35) found an increase in thrombin and MMP activity in bronchoalveolar lavage fluid of mice after MV which was not observed in C3-null mice or when wild-type mice were pretreated with a C3 inactivator. In human, polymorphisms in mannose-binding lectin 2, from the lectin-induced complement pathway, inducing a reduction of its expression were associated with lower ventilation duration and a decreased risk to develop BPD (20). A study by Tsai et al. (36) investigating cytokine and complement kinetics in patients undergoing esophageal surgery showed an increase in IL-6 immediately after surgery and an increase in the complement protein C3a only $2 \mathrm{~d}$ after the end of surgery. Together, these studies are in accordance with our findings, showing that many factors of the complement complex, which have been linked to ventilator-induced lung injury and BPD, showed a prolonged or delayed upregulation and could constitute other interesting targets for treatment and/or prevention of BPD.

The step-by-step analysis between treatment groups highlighted a leading role for LPS in the effects obtained. Indeed, a
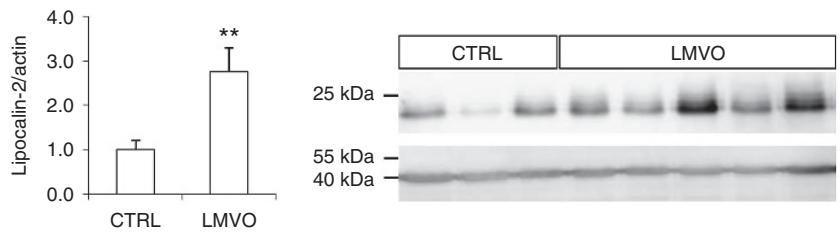

b
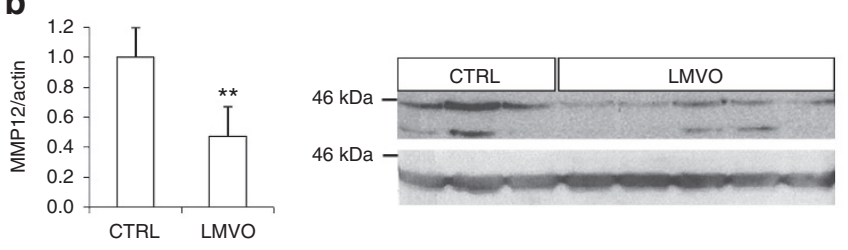

C
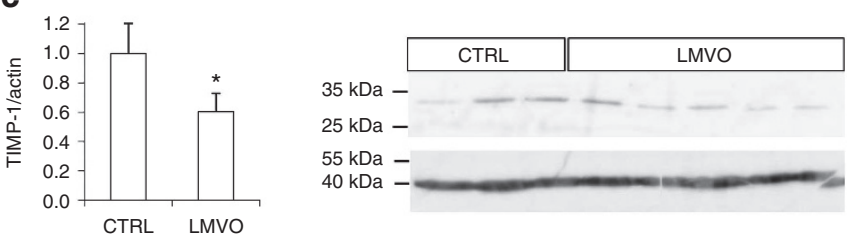

d
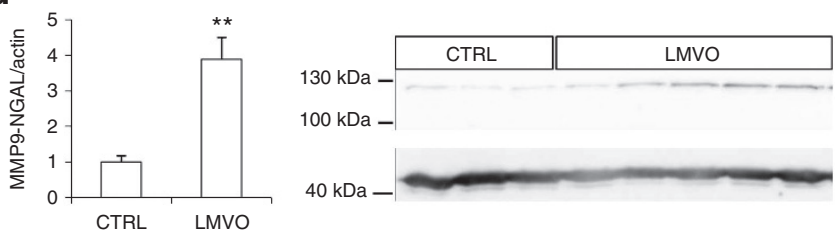

Figure 4. Protein expression of candidate genes. Four genes were also examined at the protein level by western blot, between lung samples of the LMVO $(n=5)$ vs. the CTRL $(n=3)$ group. (a) lipocalin 2, (b) MMP12, (c) TIMP1, (d) MMP9-NGAL. The expression of each protein of interest is normalized by actin protein expression. Quantification was made on three western blots and presented as mean \pm SEM. (LMVO: LPS + mechanical ventilation $+60 \%$ oxygen). ${ }^{*} P<0.05 ;{ }^{* *} P<0.01$ as analyzed by Student's $t$-test against the CTRL value.

195 genes showed an altered mRNA expression after LPS, compared to 17 and 11 after additional ventilation and oxygen treatment, respectively. This occurred despite the reduction of the amount of LPS of 33\% compared to the dose used in three previous similar studies $(4,7,9)$. This strong effect of LPS is even more astonishing, as these three studies showed that $24 \mathrm{~h}$ after the LPS injection (i.e., at the beginning of MV), the proinflammatory cytokine production was almost back to normal and the morphological aspect of the lung was comparable to the CTRL animals. However, as the primary goal of this particular animal model is the analysis of the combined effect of the major risk factors for lung development impairment and $\mathrm{BPD}$, we prioritized the direct comparison between the full treatment (LMVO) and the control (CTRL) group.

In summary, these gene array experiments identified mainly tissue modeling/wound repair and immune response pathways as being de novo or persistently modified $48 \mathrm{~h}$ after respiratory support. At this time point of recovery, mainly MMPs and complement factors show an altered expression pattern and may have an impact on lung development and the progression toward BPD. The implication of MMPs in many different pathways may indicate that their impact on lung 
Table 4. Primers used for the quantitative RT-PCR experiments

\begin{tabular}{lll}
\hline Primers & \multicolumn{1}{c}{ Forward } & \multicolumn{1}{c}{ Reverse $^{\prime}$} \\
\hline MMP12 & $5^{\prime}$-CGTCTCGATGTGGAGTGCCTGA-3' & $5^{\prime}$-CCGCACGCTTCATGTCTGGAGT-3' \\
S100A8 & $5^{\prime}$-TGAGTGCCCTCAGTTTGTGCAGAA-3' & $5^{\prime}$-GCTGCCACGCCCACCCTTATC-3' \\
RETNLA & $5^{\prime}$-ACAGGATCTCAGCTGGAGCGCA-3' & $5^{\prime}$-TCCGCCTTGTTCAGGGTGTCAGG-3' \\
LCN2 & $5^{\prime}$-CTGCCCTGCTGGGATGACTGCT-3' & $5^{\prime}$-GTTGGCAGCAGCGGGCATAGG-3' \\
MMP8 & $5^{\prime}$-GGCAGCGAATGCGGTCCAGAAA-3' & $5^{\prime}$-TGGCCCCTGACGAGGATGGAAG-3' \\
NOX4 & $5^{\prime}$-CCCTCCCAGTGCCTCCAGAACA-3' & $5^{\prime}$-CTTGCAGACCGGAACTGATTGCT-3' \\
TIMP1 & $5^{\prime}$-CTGCCCACTTGGTGAACGCCC-3' & $5^{\prime}$-ACCTGTCAGGCCCGGAACAGT-3' \\
MMP9 & $5^{\prime}$-TCCCAGAACCGCAGCGAGGA-3' & $5^{\prime}$-ACTGTGCACACCCCACAGCC-3' \\
CAMP & $5^{\prime}$-GTCCAGACCAAGGGTACAGC-3' \\
CHI3L1 & $5^{\prime}$-AGGGGAGTCCTCGTGGTAGT-3' \\
CHIA & $5^{\prime}$-TGCAACCCCATAGGGACGTCC-3' & $5^{\prime}$-GCTGAGGGTCTGGGAAACGGC-3' \\
\hline
\end{tabular}

development exceeds by far their role just as modulators of the ECM. Detailed studies will be essential to further explore their potential as targets for the prevention or treatment of BPDassociated lung development arrest.

\section{METHODS}

\section{Animal Procedures}

Pregnant Wistar rats (Charles River Laboratories, L'Arbresle Cedex, France) were housed in a controlled $12 \mathrm{~h}$ light-dark cycle room, with food and water ad libitum. Litter size was reduced to 8-10 pups at postnatal day 1 (P1) and kept constant during the experiment. Pups were marked and weighted every day, and males were randomly assigned to one of four groups: the controls (CTRL), the LPS-injected group (LPS), the group with LPS-injection and $6 \mathrm{~h}$ of MV with room air (LMV), and the group of LPS-injection and $6 \mathrm{~h}$ of MV with $60 \%$ oxygen (LMVO). All animals except controls received an i.p. injection of $2 \mathrm{mg} / \mathrm{kg}$ freshly prepared LPS E. coli serotype 026:B6 (Sigma-Aldrich, St Louis, MO) $24 \mathrm{~h}$ before MV. CTRLs were injected the same volume of $\mathrm{NaCl} 0.9 \%$. At P5 or P6, pups of LMV and LMVO groups were anesthetized with a mixture of ketamine $38 \mathrm{mg} / \mathrm{kg}$ (Ketanarkon 100; Streuli, Uznach, Switzerland), acepromazine $0.76 \mathrm{mg} / \mathrm{kg}$ (Prequillan; Arovet AG, Zollikon, Switzerland), and atropine $0.06 \mathrm{mg} / \mathrm{kg}$ (Hanseler AG, Herisau, Switzerland). After an orotracheal intubation by direct laryngoscopy with a $24 \mathrm{G}$ i.v. catheter (Optiva W I.V.Catheter; Smiths Medical International, Rossendale, UK), the tube was connected to a rodent ventilator (Voltek Rodent Ventilator; Voltek Enterprises, Toronto, Canada) in volume-controlled mode with a tidal volume of $15 \mathrm{ml} / \mathrm{kg}$, a respiratory rate of $25 / \mathrm{min}$, and a inspiratory/expiratory time ratio of 1:3. Anesthesia was maintained by adding 1-2\% of isoflurane (Provet AG, Lyssach, Switzerland) to the inspiratory circuit using a Combi-vet evaporator (Rothacher and Partner Electronics, Bern, Switzerland). During the entire experiment, heart rate (Hewlett-Packard, Dubendorf, Switzerland) was continuously monitored. Ventilated animals were kept on a heated blanket and maintained under a custom-made cover. Body temperature was measured with a rectal probe connected to a digital thermometer (Dynatime SA, La Chaux-de-Fonds, Switzerland) and remained quite stable at $35 \pm 1{ }^{\circ} \mathrm{C}$ during the whole experiment. Nonventilated animals were set aside in a small box, covered with tissues, and body temperature was maintained within the same limits with a heating lamp. Fluid was supplied after $3 \mathrm{~h}$ of ventilation by i.p. injection of Ringer's lactate $10 \mathrm{ml} / \mathrm{kg}$ (Ri-lac; B. Braun Medical AG, Sempach, Switzerland). After $5 \frac{1 / 2}{2}$ of MV, isoflurane was reduced in 2 to 3 steps over $30 \mathrm{~min}$ from baseline treatment $(1-2 \%)$ to reach $0 \%$ at the end of the 6 -h ventilation period. Ventilation parameters and oxygen were also reduced then, and the rat pups extubated as soon as spontaneous breathing reappeared, normally within minutes. Animals were returned to their mother when completely awake. Animals from the CTRL and LPS groups were also separated during $6 \mathrm{~h}$ from their mother by maintaining their body temperature at $34-36^{\circ} \mathrm{C}$, and they received one i.p. injection of Ringer lactate, too.

In preliminary experiments, the effect of MV on blood gases and the acid/base status of the animals were analyzed by performing arterial blood gas measurements. After either 1 or $6 \mathrm{~h}$ of MV, the carotid artery was prepared, cut, and the arterial blood collected into a capillary for blood gas measurement on a handheld blood gas analyzer (i-Stat 1; Abbott Point of Care, Princeton, NJ).

\section{Institutional Review}

All animal procedures were conducted according to the guidelines of the "Swiss Legislation for Animal Protection" act. The detailed protocol was approved by the responsible Veterinary Commission of the Canton of Vaud (authorization number: 1943.1).

\section{Preparation of Lung Tissue and RNA Extraction}

Lung harvest and preparation was done as described previously (7). Total lung RNA was extracted with TRIzol (Invitrogen, Carlsbad, CA) following the standard protocol. After the extraction step, the supernatant was recovered, an equal volume of $\mathrm{EtOH} 70 \%$ was added and the mix loaded onto RNeasy mini columns (Qiagen, Hilden, Germany). RNA was extracted according the Qiagen protocol.

\section{Affymetrix Gene Array}

Total RNA from nine rat lungs per group were used and pooled by three, leading to three gene arrays per group. All RNA quantities were assessed by NanoDropND-1000 spectrophotometer, and the RNA quality was assessed using RNA 6000 NanoChips with the Agilent 2100 Bioanalyzer (Agilent, Palo Alto, CA). For each sample, $100 \mathrm{ng}$ of total RNA were amplified using the Ambion WT Expression Kit (4411973; Life Technologies, Lucerne, Switzerland). An amount of 5.5 $\mu \mathrm{g}$ cDNA was fragmented and labeled with GeneChip WT Terminal Labeling kit (901525; Affymetrix, Santa Clara, CA). Affymetrix rat gene 1.0ST arrays were hybridized with $2.3 \mu \mathrm{g}$ of fragmented target, at $45{ }^{\circ} \mathrm{C}$ for $16 \mathrm{~h}$, washed, and stained according to the protocol described in Affymetrix GeneChip Expression Analysis Manual (Fluidics protocol FS450_0007).

The arrays were scanned with the GeneChip Scanner 3000 7G (Affymetrix). Normalized expression signals were calculated from Affymetrix CEL files by the Robust Multi-array Average algorithm, using the Affymetrix Expression Console Software. Hybridization quality was assessed using Expression Console Software as well. All statistical analyses were performed using the free high-level interpreted statistical language R. Differential hybridized features were 
identified using the R Bioconductor package "limma" that implements linear models for microarray data (37). The $P$ values were adjusted for multiple testing with Benjamini and Hochberg's method to control the false discovery rate (38). Probe sets showing an false discovery rate $<0.05$ were considered significant. Metacore software from Genego was used to assess the different pathways and processes affected by the gene expression changes obtained in the gene arrays.

\section{Quantitative RT-PCR}

Using Prime Script 1st strand cDNA synthesis kit (Takara BIO, Otsu, Japan), 1- $\mu$ g RNA was reverse-transcribed. Of the resulting cDNA, $50 \mathrm{ng}$ was amplified using the Absolute qPCR SYBR Green mix (ABgene, Epsom, UK): $15 \mathrm{~min}$ at $95{ }^{\circ} \mathrm{C}$ and then 45 cycles of $15 \mathrm{~s}$ at $95{ }^{\circ} \mathrm{C}, 30 \mathrm{~s}$ at $60{ }^{\circ} \mathrm{C}$, and $30 \mathrm{~s}$ at $72{ }^{\circ} \mathrm{C}$ on a Rotor-Gene 600 PCR equipment. Analysis of the results was made with the Rotor-Gene 600 Series Software 1.7 (Qiagen). Sequences of primers used are summarized in Table 4.

\section{Western Blot}

Lung tissue was extracted in a lysis buffer composed of: $10 \mathrm{mmol} / \mathrm{l}$ HEPES, $10 \mathrm{mmol} / 1 \mathrm{KCl}, 0.1 \mathrm{mmol} / \mathrm{l} \mathrm{EGTA}, 1 \mathrm{mmol} / \mathrm{l}$ dithiothreitol, 0.5 $\mathrm{mmol} / \mathrm{l} \mathrm{PMSF}$, and $0.6 \% \mathrm{NP}-40$. Antiproteases were added just before use. Proteins were loaded on 7.5, 10, or $12.5 \%$ acrylamide gels, depending on the size of the protein of interest. After migration, proteins were transferred on polyvinylidene difluoride membranes (Bio-Rad Laboratories, Hercules, CA). Antibodies used for the hybridizations were: anti-actin (20-33) monclonal rabbit antibody (Sigma-Aldrich, Darmstadt, Germany), rat lipocalin-2/NGAL antibody (AF3508; RnD Systems, Minneapolis, MN), TIMP1(H-150) antibody (sc-5538; Santa Cruz Biotechnology, Dallas, TX), rabbit monoclonal anti-MMP12 (C-terminus) antibody (clone EP1261Y) (MABT147; Merck Millipore, Darmstadt, Germany), and anti-MMP9 antibody [56-2A4] (ab58803; Abcam, Cambridge, UK). For some of them, the Vectastain ABC kit was used to enhance the signal (Vector Laboratories, Burlingame, CA). Western blots were quantified using ImageJ software. A rectangle with a defined surface was used to measure the integrated density signal in the same rectangle for each lane. Expression of the protein of interest was then normalized by the actin protein expression.

\section{SUPPLEMENTARY MATERIAL}

Supplementary material is linked to the online version of the paper at http:// www.nature.com/pr

\section{ACKNOWLEDGMENTS}

We thank Keith Harshmann, Sylvain Pradervand, and Otto Hagenbuchle of the Genomic Technologies Facility of the Center for Integrative Genomics of the University of Lausanne for technical support regarding gene array utilization and statistical analysis of the results and Sam Vasilevsky for the critical revision of the manuscript.

\section{STATEMENT OF FINANCIAL SUPPORT}

This study was supported by the Swiss National Science Foundation (Projects: $310030-118238$ and 310030-125397) and by the Wyeth Foundation for the health of children and adolescents, Switzerland.

Disclosure: The authors declare that they have no financial or other competing interests.

\section{REFERENCES}

1. Bäckström E, Lappalainen U, Bry K. Maternal IL-1beta production prevents lung injury in a mouse model of bronchopulmonary dysplasia. Am J Respir Cell Mol Biol 2010;42:149-60.

2. Coalson JJ. Pathology of bronchopulmonary dysplasia. Semin Perinatol 2006;30:179-84.

3. Bourbon J, Boucherat O, Chailley-Heu B, Delacourt C. Control mechanisms of lung alveolar development and their disorders in bronchopulmonary dysplasia. Pediatr Res 2005;57(5 Pt 2):38R-46R.

4. Kroon AA, Wang J, Huang Z, Cao L, Kuliszewski M, Post M. Inflammatory response to oxygen and endotoxin in newborn rat lung ventilated with low tidal volume. Pediatr Res 2010;68:63-9.
5. Mokres LM, Parai K, Hilgendorff A, et al. Prolonged mechanical ventilation with air induces apoptosis and causes failure of alveolar septation and angiogenesis in lungs of newborn mice. Am J Physiol Lung Cell Mol Physiol 2010;298:L23-35.

6. Hilgendorff A, Parai K, Ertsey R, et al. Inhibiting lung elastase activity enables lung growth in mechanically ventilated newborn mice. Am J Respir Crit Care Med 2011;184:537-46.

7. Trummer-Menzi E, Gremlich S, Schittny JC, et al. Evolution of gene expression changes in newborn rats after mechanical ventilation with reversible intubation. Pediatr Pulmonol 2012;47:1204-14.

8. Nagato AC, Bezerra FS, Lanzetti M, et al. Time course of inflammation, oxidative stress and tissue damage induced by hyperoxia in mouse lungs. Int J Exp Pathol 2012;93:269-78.

9. Roth-Kleiner M, Ridsdale R, Cao L, et al. Lipopolysaccharide exposure modifies high tidal volume ventilation-induced proinflammatory mediator expression in newborn rat lungs. Pediatr Res 2007;61:191-6.

10. Masumoto K, de Rooij JD, Suita S, Rottier R, Tibboel D, de Krijger RR. Expression of matrix metalloproteinases and tissue inhibitors of metalloproteinases during normal human pulmonary development. Histopathology 2005;47:410-9.

11. Tambunting F, Beharry KD, Hartleroad J, Waltzman J, Stavitsky Y, Modanlou HD. Increased lung matrix metalloproteinase-9 levels in extremely premature baboons with bronchopulmonary dysplasia. Pediatr Pulmonol 2005;39:5-14.

12. Wang $Z$, Zheng $T$, Zhu Z, et al. Interferon gamma induction of pulmonary emphysema in the adult murine lung. J Exp Med 2000;192:1587-600.

13. Harijith A, Choo-Wing R, Cataltepe S, et al. A role for matrix metalloproteinase 9 in IFN $\gamma$-mediated injury in developing lungs: relevance to bronchopulmonary dysplasia. Am J Respir Cell Mol Biol 2011;44:621-30.

14. Buckley S, Warburton D. Dynamics of metalloproteinase-2 and -9, TGFbeta, and uPA activities during normoxic vs. hyperoxic alveolarization. Am J Physiol Lung Cell Mol Physiol 2002;283:L747-54.

15. Chetty A, Cao GJ, Severgnini M, Simon A, Warburton R, Nielsen HC. Role of matrix metalloprotease-9 in hyperoxic injury in developing lung. Am J Physiol Lung Cell Mol Physiol 2008;295:L584-92.

16. Sweet DG, Curley AE, Chesshyre E, et al. The role of matrix metalloproteinases -9 and -2 in development of neonatal chronic lung disease. Acta Paediatr 2004;93:791-6.

17. Ekekezie II, Thibeault DW, Simon SD, et al. Low levels of tissue inhibitors of metalloproteinases with a high matrix metalloproteinase-9/ tissue inhibitor of metalloproteinase-1 ratio are present in tracheal aspirate fluids of infants who develop chronic lung disease. Pediatrics 2004;113:1709-14.

18. Triebel S, Bläser J, Reinke H, Tschesche H. A $25 \mathrm{kDa}$ alpha 2-microglobulin-related protein is a component of the $125 \mathrm{kDa}$ form of human gelatinase. FEBS Lett 1992;314:386-8.

19. Yan L, Borregaard N, Kjeldsen L, Moses MA. The high molecular weight urinary matrix metalloproteinase (MMP) activity is a complex of gelatinase B/MMP-9 and neutrophil gelatinase-associated lipocalin (NGAL). Modulation of MMP-9 activity by NGAL. J Biol Chem 2001;276:37258-65.

20. Capoluongo E, Vento G, Rocchetti S, et al. Mannose-binding lectin polymorphisms and pulmonary outcome in premature neonates: a pilot study. Intensive Care Med 2007;33:1787-94.

21. Inoue $\mathrm{H}$, Ohga $\mathrm{S}$, Kusuda $\mathrm{T}$, et al. Serum neutrophil gelatinase-associated lipocalin as a predictor of the development of bronchopulmonary dysplasia in preterm infants. Early Hum Dev 2013;89:425-9.

22. Lafuma C. [Elastases and pulmonary pathologies]. Pathol Biol (Paris) 1988;36:1124-32.

23. Sandhaus RA, Turino G. Neutrophil elastase-mediated lung disease. COPD 2013;10:Suppl 1:60-3.

24. Kawabata K, Hagio T, Matsuoka S. The role of neutrophil elastase in acute lung injury. Eur J Pharmacol 2002;451:1-10.

25. Hilgendorff A, Parai K, Ertsey R, et al. Neonatal mice genetically modified to express the elastase inhibitor elafin are protected against the adverse effects of mechanical ventilation on lung growth. Am J Physiol Lung Cell Mol Physiol 2012;303:L215-27. 
26. Kong MY, Li Y, Oster R, Gaggar A, Clancy JP. Early elevation of matrix metalloproteinase- 8 and -9 in pediatric ARDS is associated with an increased risk of prolonged mechanical ventilation. PLoS One 2011;6: e22596.

27. Cederqvist $\mathrm{K}$, Janer J, Tervahartiala $\mathrm{T}$, et al. Up-regulation of trypsin and mesenchymal MMP-8 during development of hyperoxic lung injury in the rat. Pediatr Res 2006;60:395-400.

28. Albaiceta GM, Gutierrez-Fernández A, García-Prieto E, et al. Absence or inhibition of matrix metalloproteinase- 8 decreases ventilator-induced lung injury. Am J Respir Cell Mol Biol 2010;43:555-63.

29. Greenlee KJ, Werb Z, Kheradmand F. Matrix metalloproteinases in lung: multiple, multifarious, and multifaceted. Physiol Rev 2007;87:69-98.

30. Page-McCaw A, Ewald AJ, Werb Z. Matrix metalloproteinases and the regulation of tissue remodelling. Nat Rev Mol Cell Biol 2007;8:221-33.

31. Khokha R, Murthy A, Weiss A. Metalloproteinases and their natural inhibitors in inflammation and immunity. Nat Rev Immunol 2013;13:649-65.

32. Löffek S, Schilling O, Franzke CW. Series "matrix metalloproteinases in lung health and disease": biological role of matrix metalloproteinases: a critical balance. Eur Respir J 2011;38:191-208.
33. Rodríguez D, Morrison CJ, Overall CM. Matrix metalloproteinases: what do they not do? New substrates and biological roles identified by murine models and proteomics. Biochim Biophys Acta 2010;1803: 39-54.

34. Liu K, Mao YF, Zheng J, et al. SC5b-9-induced pulmonary microvascular endothelial hyperpermeability participates in ventilator-induced lung injury. Cell Biochem Biophys 2013;67:1421-31.

35. Takahashi K, Saha D, Shattino I, et al. Complement 3 is involved with ventilator-induced lung injury. Int Immunopharmacol 2011;11:2138-43.

36. Tsai JA, Lund M, Lundell L, Nilsson-Ekdahl K. One-lung ventilation during thoracoabdominal esophagectomy elicits complement activation. J Surg Res 2009;152:331-7.

37. Smyth GK. Limma: linear models for microarray data. In: Gentleman R, Carey V, Huber W, Irizarry R, Dudoit S, eds. Bioinformatics and Computational Biology Solutions Using R and Bioconductor. New York: Springer, 2005:397-420.

38. Benjamini Y, Hochberg Y. Controlling the false discovery rate: a practical and powerful approach to multiple testing. J R Stat Soc Series B 1995;57:289-300. 